\title{
ON HAUSDORFF COMPACTIFICATIONS OF NON-LOCALLY COMPACT SPACES
}

\section{JAMES HATZENBUHLER and DON A. MATTSON}

\author{
Department of Mathematics \\ Moorhead State University \\ Moorhead, Minnesota 56560
}

(Received December 19, 1978 and in Revised form February 2, 1979)

ABSTRACT. Let $\mathrm{X}$ be a completely regular, Hausdorff space and let $\mathrm{R}$ be the set of points in $X$ which do not possess compact neighborhoods. Assume $\mathrm{R}$ is compact. If $\mathrm{X}$ has a compactification with a countable remainder, then so does the quotient $X / R$, and a countable compactification of $X / R$ implies one for $X-R$. A characterization of when $X / R$ has a compactification with a countable remainder is obtained. Examples show that the above implications cannot be reversed.

KEY WORDS AND PHRASES. Countable remainders, compactifications, non-locally compact spaces, components of $\beta X-X$.

1980 Mathematics Subject Classification Codes: 54035. 


\section{INTRODUCTION.}

Let $\mathrm{X}$ be a completely regular, Hausdorff topological space. The question of characterizing when $X$ has a Hausdorff compactification $\alpha X$, where $\alpha X-X$ is countably infinite, has been answered for the locally compact case by Magill [2] and for the case when $\alpha X=\beta X$ by Okuyama [4] (where $\beta X$ is the Stone-Cech compactification of $\mathrm{X}$ ). In case $\mathrm{X}$ is an arbitrary completely regular space, no such characterization has been given. The purpose of this paper is to contribute results toward such a characterization.

Let $R$ be the set of points in $X$ which do not possess compact neighborhoods. Then for all compactifications $\alpha \mathrm{X}$ of $\mathrm{X}, \mathrm{R}=\mathrm{C} 1_{\alpha \mathrm{X}}(\alpha \mathrm{X}-\mathrm{X}) \cap \mathrm{X}$. (See [5].) Herein we observe that for compact $R$, a necessary condition for $X$ to have a countable compactification is that $X / R$ have one. The main theorem of this paper characterizes when $X / R$ has a countable compactification.

\section{CHARACTERIZATION OF $\alpha(\mathrm{X} / \mathrm{R})$.}

Throughout this paper all compactifications are Hausdorff compactifications. Let $\mathrm{N}$ denote the natural numbers. If $\mathrm{R}$ is a compact, non-empty subset of a completely regular space $X$ and if $X$ has a coutable compactification $\gamma X$, then a countable compactification of $X / R$ can be obtained from $\gamma X$ by identifying $R$ to a single point. It is readily verified that the resulting space is Hausdorff.

If $\alpha(X / R)$ is a countable compactification of $X / R$, then $\alpha(X / R)$ is also a countable compactification of $x-R$. Thus, we have the following:

THEOREM 1. If $X$ is completely regular and $R$ is compact, then each of the following conditions implies the next:

(A) $\mathrm{X}$ has a countable compactification;

(B) $X / R$ has a countable compactification; 
(C) $X-R$ has a countable compactification.

Examples will be provided to show that none of these Implications can be reversed.

If $R$ is non-compact, then (A) no longer implies (C) as in Theorem 1. Let $X$ be the unit disc in the standard plane with a countable dense subset removed from the boundary. The remaining boundary points constitute $R$. Then, clearly, $X$ has a countable compactification but $X-R$, the open disc, has no countable compactification.

Let $Y=(\beta X-X) \cup R$.

THEOREM 2. Let $X$ be a completely regular Hausdorff space with $R$ compact and non-empty. Then the following are equivalent:

(A) $X / R$ has a countable compactification.

'B) $R$ is a $G_{\delta}$ set in $Y$ and components of $R$ are components of $Y$.

PROOF. (A) implies (B). Take $\left\{p_{n} \mid n \varepsilon N\right\}=\gamma(X / R)-X / R$, where $\gamma(X / R)$ is a countable compactification of $X / R$, and let $t_{0}$ be the canonical mapping of $X$ into $\gamma(X / R)$. Then $t_{0}$ has an extension $t$ which maps $B X$ onto $\gamma(X / R)$. We first show that $t$ carries $\beta X-X$ onto $\gamma(X / R)-X / R$. Since the restriction of $t$ to $X-R$ is a homeomorphism and $X-R$ is dense in $B X$ and in $\gamma(X / R)$, $t$ carries $Y$ onto $[\gamma(X / R)-X / R] \bigcup\{r\}$, where $r=t[R]$ (cf. Lemma 6.11 [1]). If $x \in R$ and $y \varepsilon-\beta X-X$, then since $R$ is compact there exists a compact neighborhood $N_{R}$ of $R$ in $\beta X$ such that $y \notin N_{R}$. Set $N=N_{R} \cap x$. Since $R \subseteq N, t_{0}[N]$ is a neighborhood of $t(x)=r$ in $x / R$. Thus, there is a neighborhood $G$ in $\gamma(x / R)$ for which $t_{0}[N]=G \cap x / R$. If $\mathrm{N}_{\mathrm{y}}$ is any neighborhood of $\mathrm{y}$ in $\beta \mathrm{X}$, choose $\mathrm{z} \varepsilon \mathrm{N}_{\mathrm{y}} \cap(\mathrm{X}-\mathrm{N})$. Then $t(z) \notin G$ and $i t$ follows from the continuity of $t$ that $t(x) \neq t(y)$. Hence $t[\beta X-X]=\gamma(X / R)-X / R$. 
Next, let $K_{n}=t^{-1}\left(p_{n}\right)$, for each $n \varepsilon N$. Evidently, $\beta X-X=\bigcup\left\{K_{n} \mid n \varepsilon N\right\}$. Since each $K_{n}$ is compact, the sets $Y-K_{n}$ are open in $Y$ and $R=\bigcap\left\{Y-K_{n} \mid n \in N\right\}$. Thus $R$ is a $G_{\delta}$-set in $Y$.

Let $C$ be a component of $R$ and let $C_{1}$ be a component of $Y$, where $C_{-} C_{1}$. If $C \neq C_{1}$, choose $x \in C_{1}-C$. Now there exists a continuous injection $f$ of $\left\{p_{n} \in N\right\} \quad\{r\}$ into the real numbers. (See [3]). But $f \circ t C_{1}$ must be connected and not a singleton, since $t[R] \neq t(x)$. This contradicts the fact that the image of $f$ is countable. Thus, $C=C_{1}$, so that components of $R$ are components of $\mathrm{Y}$.

(B) implies (A). First we show that there exist sets $\left\{U_{n} \mid n \in N\right\}$ which are clopen in $Y$ such that $\bigcap\left\{U_{n} \mid n \varepsilon N\right\}=R$. Note that $Y$ is compact. Let $\left\{V_{n} \mid n \in N\right\}$ be open subsets of Y satisfying $\bigcap\left\{V_{n} \mid n \in N\right\}=R$. For each $n \in N$, set $K_{n}=Y-V_{n}$. We assume that each $K_{n} \neq \emptyset$. Let $(x, r) \varepsilon K_{n} \times R$. Since $x$ and $r$ are in distinct quasi-components of $Y$, there exists a clopen neighborhood $W_{n}(x, r)$ of $r$ in $Y$, where $x \notin W_{n}(x, r)$. Now $\left\{W_{n}(x, r) \mid r \in R\right\}$ is an open covering of $R$ so that a finite subfamily $\left\{w_{n}\left(x, r_{1}\right) \mid 1=1, \ldots, p(x)\right\}$ covers $R$. Take $w_{n}(x)=\bigcup\left\{w_{n}\left(x, r_{1}\right) \mid 1=1, \ldots, p(x)\right\}$. Thus $W_{n}(x)$ is a clopen subset of $Y . R \subseteq W_{n}(x)$, and $x \notin W_{n}(x)$. Since $\left\{Y-W_{n}(x) \mid x \in K_{n}\right\} \quad$ is an open cover of $K_{n}$, there is a finite subcover $\left\{Y-W_{n}\left(x_{j}\right) \mid j=1, \ldots, q(n)\right\}$.

For each $n \in N$, let $U_{n}=\bigcap\left\{w_{n}\left(x_{j}\right) \mid j=1, \ldots, q(n)\right\}$. Then each $U_{n}$ is a clopen subset of $Y, R \subseteq U_{n}$ and $K_{n} \subseteq Y-U_{n}$. Hence $R=\bigcap\left\{U_{n} \mid n \varepsilon N\right\}$. Let $C_{1}=Y-U_{1}$, and for $n>1$, take $C_{n}=\left[Y-\bigcap\left\{U_{1} \mid 1=1, \ldots, n\right\}\right]-$ $U\left\{c_{1} \mid 1=1, \ldots, n-1\right\}$. Then each $c_{n}$ is a clopen subset of $Y$ and $\beta X-X=$ $\bigcup\left\{C_{n} \mid n \in N\right\}$.

Let $\sim$ be the equivalence relation in $\beta X$ which identifies each $C_{n}$ to a point and $R$ to a point. The projection of $B X$ onto $B X / \sim$ is denoted by $\Pi$. 
For each $n \varepsilon N$, consider the point $\Pi\left[C_{n}\right]$ in $B X / \sim$. Now $\left\{C_{n}, Y-C_{n}\right\}$ is a partition of $Y$ into disjoint open sets. Thus, $C_{n}$ and $Y-C_{n}$ can be separated by open sets $U$ and $V$ in $\beta X$. Evidently, $\Pi[U]$ and $\Pi[V]$ are disjoint open subsets of $B X / \sim$. This shows that $\pi\left[C_{n}\right]$ can be separated from any other point of $\beta X / \sim$. Since points of $\beta X-Y$ have compact $\beta X-$ neighborhoods in $\beta X-Y$, it follows that $B X / \sim$ is a compact Hausdorff space.

It remins to show that $X / R$ can be embedded in $B X / \sim$ in the desired manner. Let $i$ be the natural embedding of $X$ in $\beta X$ and let $p$ be the projection of $X$ onto $X / R$. Since 1 is relation preserving, a continuous mapping $f$ of $X / R$ into $B X / \sim$ is induced such that $j \circ p=\pi \circ 1$. It follows that $j$ is also a closed mapping, hence an embedding of $X / R$ into $B X / \sim$ as desired. This completes the proof.

In [2] Magill shows that a locally compact space $X$ has a countable compactification if and only if $B X-X$ has infinitely many components. As an application of the proof of Theorem 2, the following is proven.

COROLLARY 3. Let $X$ be completely regular with $R$ compact. If $X$ has a countable compactification, then $B X-X$ has infinitely many components.

PROOF. Let $t$ be a continuous mapping of $\beta X$ onto $\alpha(X / R)$ which carries $\beta X-X$ onto $\alpha(X / R)-X / R$. Since the subspace $K=(\alpha(X / R)-X / R) U\{t(R)\}$ is compact and countable, it contains an open countable discrete subspace. Since $\alpha(X / R)-X / R$ contains infinitely many components of $K, Y$ must contain Infinitely many components.

The converse of Corollary 3 is false when $X$ is not locally compact. Example (A) shows that $X / R$ can have a countable compactification, so that $\beta X$ - $X$ has infinitely many components, but $X$ has no countable compactification. Example (A) also shows that condition (B) of Theorem 1 is not sufficient to insure that $X$ has a countable compactification when $R$ is compact. 
EXAMPLE (A). Let $S$ be the closed unit square in $R^{2}$, I be the unit interval, $L_{0}=I \times\{0\}$, and, for $n \in N, L_{n}=I \times\left\{\frac{1}{n+I}\right\}$. For $x=S-\bigcup_{n \in N} L_{n}$, It is clear that $X$ is not rim compact, and hence does not have a countable compactification (cf. [6]). Furthermore, $R=L_{0}$ and $S$ is a compactification of $X$. The existence of a continuous surfection from $\beta X$ onto $S$ which leaves $X$ fixed and which carries $B X-X$ onto $S-X$ guarantees that condition (B) of Theorem 2 is satisfied. Hence $X / R$ has a countable compactification.

The following example shows that for $R$ non-empty and compact the implication of (C) by (B) of Theorem 1 cannot be reversed. It suffices to exhibit $X$, with $R$ a singleton, where $X-R$ has a countable compactification but $X$ does not.

EXAMPLE (B). In the plane $R^{2}$ take $\mathrm{x}=[\{(\mathrm{x}, \mathrm{y}) \mid-1<\mathrm{x}<1 ;-1<\mathrm{y}<1\} \bigcup\{(1,0)\}]-\left\{\left(\frac{-\mathrm{n}}{\mathrm{n}+1}, 0\right) \mid \mathrm{n} \varepsilon \mathrm{N}\right\}$. Then $R=\{(1,0)\}$. Since $X$ is not rim compact, it has no countable compactification. However, a countable compactification for $X-R$ is obtained by adjoining the points $\left(\frac{-n}{n+I}, 0\right)$, for each $n \in N$, and taking the one-point compactification of the resulting space.

\section{REFERENCES}

1. Gillman, L. and Jerison, M. Rings of continous functions, The University Series in Higher Math., Princeton, N.J., 1960.

2. Magill, K. D., Jr. Countable compactifications, Canad. J. Math. 18 (1966), 616-620.

3. Mrowka, S. Continuous functions on countable subspaces, Port. Math. $\underline{29}(1970), 177-180$.

4. Okuyama, A. A characterization of a space with countable infinity, Proc. A.M.S. 28 (1971), 595-597.

5. Rayburn, M. On Hausdorff compactifications, Pac. J. of Math. 44 (1973), 707-714.

6. Zippin, L. On semicompact spaces, Amer. J. Math. 57 (1935), 327-341. 


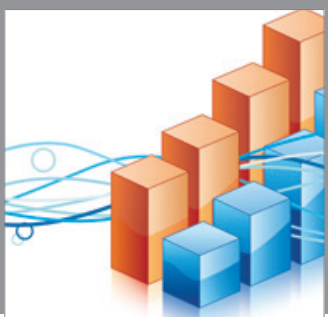

Advances in

Operations Research

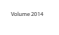

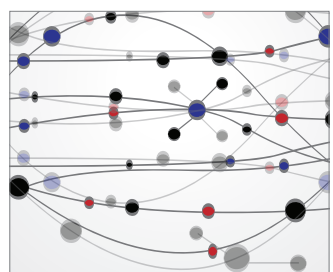

\section{The Scientific} World Journal
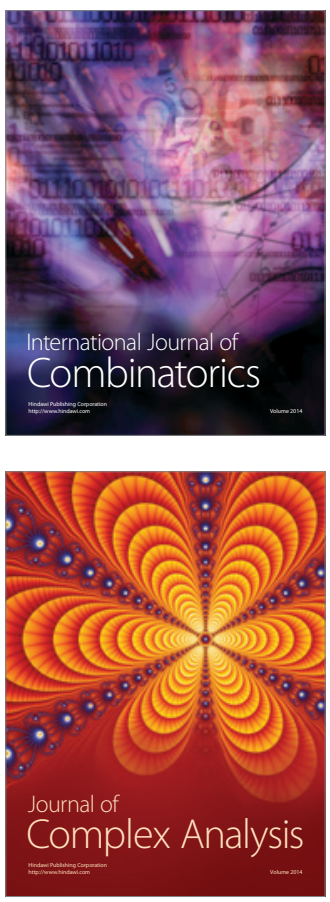

International Journal of

Mathematics and

Mathematical

Sciences
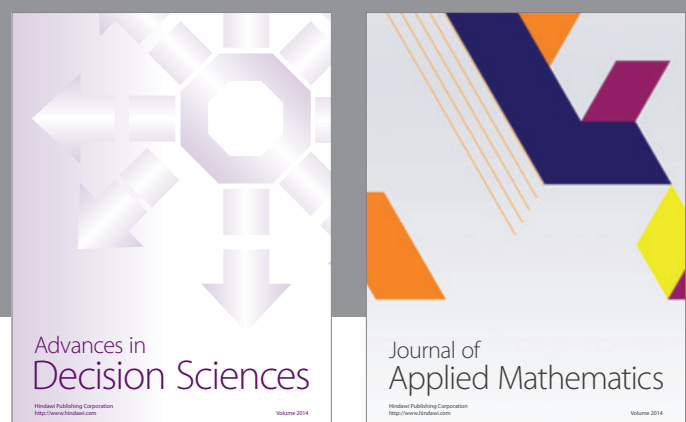

Journal of

Applied Mathematics
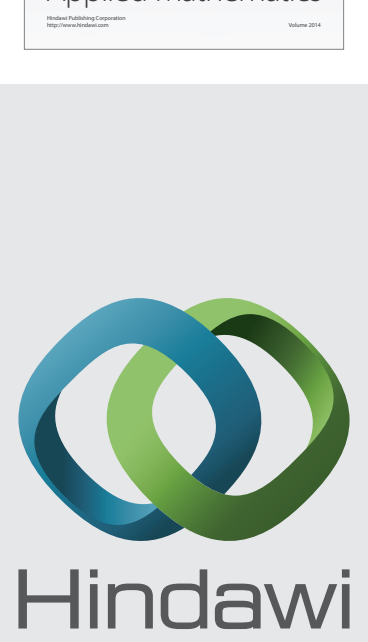

Submit your manuscripts at http://www.hindawi.com
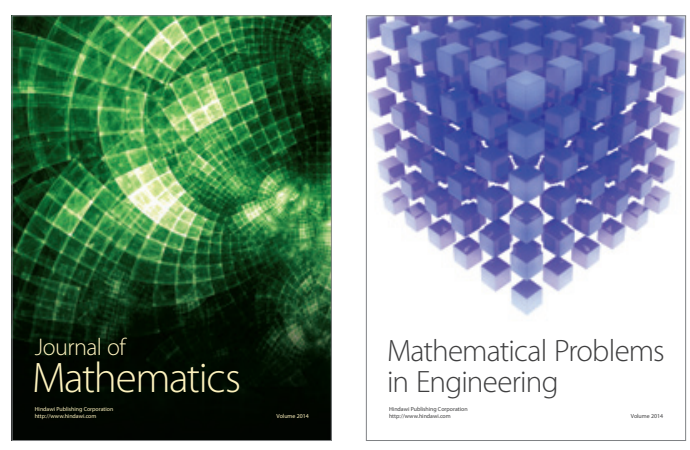

Mathematical Problems in Engineering
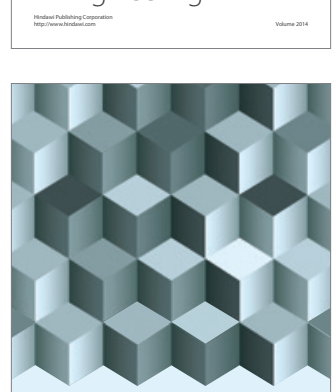

Journal of

Function Spaces
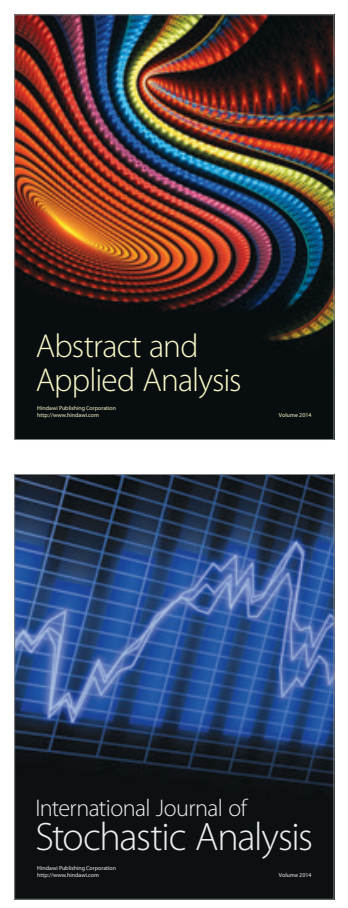

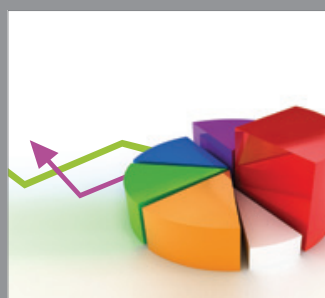

ournal of

Probability and Statistics

Promensencen
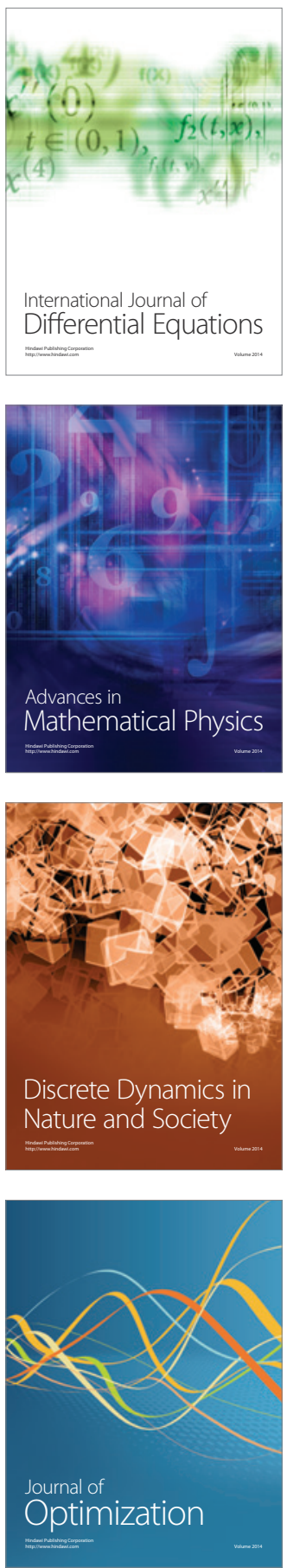